\title{
Magneto-actuated immunoassay for the detection of Mycobacterium fortuitum in hemodialysis water
}

\author{
Michelle Fernanda Brugnera ${ }^{\mathrm{a}, \mathrm{c}, 1}$, Reynaldo Bundalian Jr ${ }^{\mathrm{a}, \mathrm{d}, 1}$, Tamara Laube ${ }^{\mathrm{a}}$, Esther Julián ${ }^{\mathrm{b}}$, \\ Marina Luquin ${ }^{\mathrm{b}}$, Maria Valnice Boldrin Zanoni ${ }^{\mathrm{c}}$, Maria Isabel Pividori ${ }^{\mathrm{a}, *}$ \\ ${ }^{a}$ Grup de Sensors i Biosensors, Departament de Química, Universitat Autònoma de Barcelona, Bellaterra, Spain \\ ${ }^{\mathrm{b}}$ Departament de Genética i de Microbiologia, Facultat de Biociències, Universitat Autònoma de Barcelona, Bellaterra, Spain \\ ${ }^{\mathrm{c}}$ Department of Analytical Chemistry, Institute of Chemistry, UNESP-Univ Estadual Paulista, Araraquara, SP, Brazil \\ ${ }^{\mathrm{d}}$ Department of Chemistry, College of Arts and Sciences, Angeles University Foundation, Angeles City, Philippines
}

\section{A R T I C L E I N F O}

\section{Article history:}

Received 9 October 2015

Received in revised form

12 February 2016

Accepted 16 February 2016

Available online 18 February 2016

Keywords:

Magneto-actuated immunoassay

Immunomagnetic separation

Mycobacterium fortuitum

Hemodialysis water

\begin{abstract}
A B S T R A C T
This paper addresses a sensitive method for the detection of mycobacteria in hemodialysis water samples based on a magneto-actuated immunoassay with optical readout. In this approach, micro $(2.8 \mu \mathrm{m})$ sized magnetic particles were modified with an antibody against the lipoarabinomannan (LAM) located in the mycobacterial cell wall. The system relies on the immunocapturing of the mycobacteria with the tailored antiLAM magnetic particles to pre-concentrate the bacteria from the hemodialysis samples throughout an immunological reaction. The performance of the immunomagnetic separation on the magnetic carrier was evaluated using confocal microscopy to study the binding pattern, as well as a magneto-actuated immunoassay with optical readout for the rapid detection of the bacteria in spiked hemodialysis samples. In this approach, the antiLAM polyclonal antibody was labeled with fluorescein isothiocyanate. The optical readout was achieved by the incubation with a secondary anti-fluorescein antibody labeled with peroxidase as optical reporter. The magneto-actuated immunoassay was able to detect mycobacteria contamination in hemodialysis water at a limit of detection of $13 \mathrm{CFU} \mathrm{mL}^{-1}$ in a total assay time of $3 \mathrm{~h}$ without any previous culturing pre-enrichment step.
\end{abstract}

(c) 2016 Elsevier B.V. All rights reserved.

\section{Introduction}

The number of patients with end-stage renal disease (ESRD) receiving renal replacement therapy (RRT) was globally estimated at more than 1.4 million with $8 \%$ increase in annual incidence [1]. Much of the patients with ESRD undergo maintenance hemodialysis most especially in the absence of a compatible kidney donor. Hemodialysis is presently routinely used for the treatment of acute renal failure and end stage renal disease. The dialysis machine works by substituting the kidney function by using a semipermeable membrane to remove waste and water from the blood into the dialysate fluid [2]. Due to the exposure of dialysis patients with water, infection arising from contamination of dialysis water remains to be a significant cause of death among patients. Infection has been identified as the second leading cause of death among dialysis patients [3].

Among bacterial contaminants of hemodialysis water, recent

\footnotetext{
* Corresponding author.

E-mail address: isabel.pividori@uab.cat (M.I. Pividori).

${ }^{1}$ These authors contributed equally to this work.
}

interest has been focused on the isolation and identification of non-tuberculous mycobacteria (NTM) due to their potential health implications. Isolated NTM from hemodialysis water includes pathogenic environmental mycobacteria (PEM) such as Mycobacterium fortuitum and Mycobacterium chelonae [4]. M. fortuitum and $M$. chelonae are rapid growing environmental mycobacteria rarely associated with infections of hemodialysis patients but can cause serious dialysis related peritonitis. M. chelonae exhibits more resistance to antimicrobial agents than $M$. fortuitum but with variable antibiotic susceptibility [5]. On the other hand, $M$. fortuitum biofilm formation and resistance to disinfection has been reported in several studies [6,7].

The primary strategy in preventing infection among dialysis patients requires ensuring safe and quality water. To achieve this, good disinfection strategies are necessary which takes into account frequency of disinfection ensuring water quality. Traditional monitoring of bacterial contamination in hemodialysis water is performed by microbial cultivation; this requires growing of the microorganisms in artificial medium and identification of the isolates either with biochemical testing or PCR-based techniques. The overall sensitivity of this technique may depend on the culture medium used, the incubation temperature, and the length of 
incubation time to allow for slow-growing microbes such as mycobacteria to grow. While dialysis fluid undergoes microbiological testing as part of the routine quality assurance in production, many of the microbial contaminants may be found in the surfaces which still do not rule out the potential contamination of the fluid. In such cases, readily available information on the presence of microbial contaminants could have practical impact or the frequency of disinfection as part of the infection control measures [8]. As detection of PEM by traditional culture techniques is time consuming, laborious and inefficient, there is a need for novel rapid method to detect mycobacteria to ensure the quality in hemodialysis water sources.

Superparamagnetic iron oxide particles, with sizes ranging from a few nanometers up to micrometer dimensions have been applied in a broad variety of fields ranging from molecular imaging, drug delivery to clinical diagnostics or food safety [9]. Their functionalization with different reactive groups, especially antibodies, makes them useful to bind and pre-concentrate a variety of targets under magnetic actuation, through immunomagnetic separation. Moreover, the pre-concentration of the target from the complex sample is easily achieved, offering an attractive technology to be implemented in magneto-actuated platforms including biosensors [10], microfluidic platforms [11], among others [12].

Hence, this work proposed to develop a rapid detection method for PEM in hemodialysis water using M. fortuitum as model. Micro $(2.8 \mu \mathrm{m})$-sized magnetic particles (MPs) were firstly modified with anti-lipoarabinomannan (LAM) antibodies to capture and preconcentrate the mycobacteria from hemodialysis samples based on an immunological reaction. The antiLAM antibody was also conjugated with fluorescein for further reaction with the optical reporter antiFLU-HRP. Finally, mycobacteria detection in hemodialysis water samples is also presented as a magneto-actuated immunoassay with optical readout.

\section{Experimental section}

\subsection{Instrumentation}

Magnetic actuation was achieved with a 96-well plate magnet (Product no. 21358 Thermo Fisher Scientific, Waltham, USA). Polystyrene MaxiSorp microplates from Nunc (Catalogue no. 442404, Roskilde, DK) were used for classical ELISA. The magnetoactuated immunoassays were performed in polypropylene 96-well microtiter plates (Ref. no. 153364, Corning) while the optical readout in polystyrene 96-well microtiter plates (Ref. no. 269787, Roskilde, DK). The purification of the antiLAM-FLU antibodies was performed using ZebaTM Micro Desalt spin columns 70,000 MW cut-off (Catalogue no. 89878, Pierce Rockford, IL). The optical readout was performed on a TECAN Sunrise microplate reader with Magellan v4.0 software, and measurement were in all cases performed at $450 \mathrm{~nm}$. Confocal microscopy was done on a Leica confocal microscope, TCS SP5.

\subsection{Chemicals and biochemicals}

Primary antibody includes a polyclonal antiLAM antibody from guinea pig (Catalogue no. MBS315001, MyBiosource). The polyclonal antiLAM antibody from guinea pig was immobilized in the surface of the magnetic particles (tosyl-MPs) (Dynabeads M-280 Tosylactivated, Product no. 142.04, Dynal Biotech ASA, Oslo, Norway) and was also labeled with fluorescein isothiocyanate (FITC) from Sigma (Product no. F7250 Sigma, USA). To come up with an indirect sandwich immunoassay format, an antiFluorescein-POD secondary antibody (antiFLU-HRP) (Product no. 11426346910, Roche Diagnostics, Manheim Germany) was used, while an anti- guinea pig-HRP antibody (Product no. Ab 6771, Abcam, Cambridge, UK) were used in direct adsorption ELISA experiments.

Different sets of buffers were used for specific procedures in the experiments, and their composition is described in the supporting information. All buffers were prepared from analytical grade reagent purchased from Merck and Sigma. Reagents used for the optical measurement of the enzymatic reactions include hydrogen peroxide and TMB (3,3',5,5'-tetrametylbenzidine) solutions (TMB Substrate Kit, Ref. no. 483402, Pierce Rockford, USA). Also, a $2.0 \mathrm{M}$ solution of sulfuric acid was prepared and used to stop the TMB reaction in the magneto-actuated immunoassay experiments.

\subsection{M. fortuitum culture}

Cultivation of the $M$. fortuitum was done in trypticase soy broth (TSB) supplemented with $0.025 \%$ Tween 20 to avoid formation of clumps. Incubation was made with continuous agitation at $37^{\circ} \mathrm{C}$ and cultures were grown until the absorbance reaches approximately 0.300 A.U. with an equivalent plate colony count of $8.5 \times 10^{6}$ colony-forming units $(\mathrm{CFU}) \mathrm{mL}^{-1}$. Cultures were adjusted to approximately $1.0 \times 10^{6}$ with dilution with sterile TSB and were plated for colony counts for verification. The prepared $M$. fortuitum cultures were supplemented with $20 \%$ sterile filtered glycerol and were stored at $-20{ }^{\circ} \mathrm{C}$ until further used in the experiment.

\subsection{Immunoreactivity study of the antiLAM antibody}

In order to check the ability of antibody to detect mycobacteria, the immunoreactivity of the polyclonal antiLAM antibody from guinea pig was initially screened against pure cultures of $M$. fortuitum grown in TSB with 0,025\% Tween 20. Direct adsorption ELISA was performed using a previously reported protocol with minor modifications [13]. Briefly, $10^{6} \mathrm{CFU} \mathrm{mL}^{-1}$ of mycobacteria were harvested by centrifugation at $3500 \mathrm{~g}$ for $10 \mathrm{~min}$ [14] and were washed once with PBS and resuspended in $1 \mathrm{~mL}$ of PBS. $50 \mu \mathrm{L}$ of the mycobacterial suspension were placed and adsorbed in each well for $24 \mathrm{~h}$ at RT. The background adsorption was evaluated with blank wells prepared with $50 \mu \mathrm{L}$ of PBS. Following adsorption, the wells were washed once with $200 \mu \mathrm{L}$ of PBS. Thereafter, the plates were blocked with PBS with $1 \%$ casein at RT for $1 \mathrm{~h}$. After blocking, plates were washed three times with PBST $0.1 \%$ casein. $100 \mu \mathrm{L}$ of the antiLAM antibody $\left(1.0 \mu \mathrm{g} \mathrm{mL}^{-1}\right)$ was added and incubated for $1 \mathrm{~h}$ at RT and was followed by three washes with $200 \mu \mathrm{L}$ of PBST $0.1 \%$ casein. $100 \mu \mathrm{L}$ of the anti-guinea pig secondary antibody labeled with HRP diluted $1 / 2000$ in PBST $0.1 \%$ casein was then added, and was incubated for $30 \mathrm{~min}$. Three final washes were performed and $100 \mu \mathrm{L}$ of the TMB- $\mathrm{H}_{2} \mathrm{O}_{2}$ substrate solution was added and allowed to react under dark conditions for $30 \mathrm{~min}$. This was then followed by the addition of $100 \mu \mathrm{L}$ of $2.0 \mathrm{M}$ sulfuric acid and absorbance was measured at $450 \mathrm{~nm}$.

\subsection{Preparation of the antiLAM-FLU antibody and purification}

The antiLAM-FLU conjugates were prepared using the manufacturer's recommended protocol. Fluorescein isothiocyanate (FITC) is widely used to attach a fluorescent label to proteins via the amine group. The isothiocyanate group directly reacts with primary amines in aminoacidic residues of antibodies, mostly lysine moieties, without the need of any previous activation step. $50 \mathrm{mM}$ excess of FITC dissolved in dimethyl formamide (DMF) was added to $100 \mu \mathrm{g}$ of the polyclonal antiLAM antibody from guinea pig prepared in $450 \mu \mathrm{L}$ of the bicarbonate buffer, and was incubated at RT for $2 \mathrm{~h}$. This results in a final volume of $500 \mu \mathrm{L}$ and an antibody concentration of $200 \mu \mathrm{g} \mathrm{mL}^{-1}$. The excess FITC was 
removed by desalting the labeled antiLAM (antiLAM-FLU) in Zeba $^{\mathrm{TM}}$ Micro Desalt spin columns equilibrated with TRIS buffer solution. The number of desalting required to reduce non-specific adsorption was also optimized, as shown in the supporting information section.

\subsection{Immunoreactivity study of the antiLAM-FLU antibody}

The immunoreactivity of the antiLAM-FLU antibody was performed by direct ELISA. The wells were prepared by adsorbing $50 \mu \mathrm{l}$ of $10^{6} \mathrm{CFU} \mathrm{mL} \mathrm{m}^{-1}$ of mycobacteria in PBS at RT for $24 \mathrm{~h}$ and blocked with PBS 1\% casein. Blank wells containing PBS and later blocked with PBS $1 \%$ casein were also prepared. $100 \mu$ l of the antiLAM-FLU antibodies $\left(1.0 \mu \mathrm{L} \mathrm{mL}^{-1}\right)$ were reacted with the adsorbed mycobacteria to check the retention of the immunoreactivity following the labeling reaction. In order to check the presence of the antiLAM-FLU antibodies, two different secondary antibodies were used: anti-guinea pig-HRP antibody as well as antiFLU-HRP, in this instance to confirm the conjugation of the antibody. To achieve this, $100 \mu \mathrm{L}$ anti-guinea pig-HRP $(1 / 2000)$ or antiFLU-HRP $\left(4 \mu \mathrm{g} \mathrm{mL}^{-1}\right)$ were added to probe for the retention of immunorecognition following the modification of the antibody with FITC. The optical readout was performed as previously explained.

\subsection{Covalent immobilization of antiLAM antibody on magnetic particles and confocal microscopy study}

Covalent immobilization of the antiLAM antibodies was performed in tosyl-MPs. $100 \mu \mathrm{g}$ of the antiLAM antibodies was incubated overnight with $5 \mathrm{mg}$ of the particles in the coating buffer and was incubated at $37{ }^{\circ} \mathrm{C}$ with continuous agitation at $700 \mathrm{rpm}$ in a thermomixer. The supernatant was removed and was used to measure the percentage of antibody coupled in the particles using direct adsorption ELISA. Blocking of the unreacted tosyl groups was made with $50 \mathrm{mM}$ ethanolamine for $2 \mathrm{~h}$ with agitation. The prepared antiLAM modified MPs (antiLAM-MPs) were stored in PBS plus Tween 20 (PBST) with $0.05 \%$ sodium azide at $4{ }^{\circ} \mathrm{C}$. The antiLAM-MPs were washed twice with PBST before used in each experiment.

In order to evaluate the ability of the antiLAM-MPs in capturing mycobacteria, confocal fluorescence microscopy was performed to visualize the interaction between the antiLAM-MPs and the $M$. fortuitum. Mycobacterial DNA was stained by incubating $4.5 \mu \mathrm{L}$ of the Hoechst dye with $500 \mu \mathrm{L}$ of the mycobacteria $\left(1.0 \times 10^{5} \mathrm{CFU} \mathrm{mL}^{-1}\right)$ in TSB with $0,025 \%$ Tween 20 for $30 \mathrm{~min}$ at RT. This was followed by immunomagnetic capture of the stained mycobacteria with $200 \mu \mathrm{L}$ of the antiLAM-MP $\left(0.075 \mathrm{mg} \mathrm{mL}^{-1}\right)$ incubated for $1 \mathrm{~h}$ at RT with agitation at $750 \mathrm{rpm}$. Samples were then washed three times with PBST plus $0.1 \%$ casein. Confocal images were thereafter collected. The laser lines used were Hoechst (405 nm excitation, 415-570 nm emission) and FITC (492 nm excitation, $518 \mathrm{~nm}$ emission). Images were processed using the Imaris software.

\subsection{Optimization of the magneto-actuated immunoassay}

The magneto-actuated immunoassay for the optical detection of $M$. fortuitum was initially optimized using two-dimensional (2D) serial dilution experiments. In order to evaluate the optimal concentration of the immunoreagents, magneto-immunoassay was performed covering broad range of immunoreagent concentrations using a two-step sandwich immunoassay approach. Optimal concentrations were chosen to produce 2.0 A.U. Initially, the optimum concentration of the antiLAM-MP was evaluated using concentrations ranging from $0.100 \mathrm{mg} \mathrm{mL}^{-1}$ to $0.025 \mathrm{mg} \mathrm{mL}^{-1}$, while the concentration of the $M$. fortuitum was kept at $1.0 \times 10^{5} \mathrm{CFU} \mathrm{mL}^{-1}$. Thereafter, the optimal concentration of the antiLAM-FLU was established by analyzing $1.0 \times 10^{5} \mathrm{CFU} \mathrm{mL}^{-1}$ concentration of the mycobacteria with the optimized concentration of the antiLAM-MP while the antiFLU-HRP secondary antibody concentration was kept at $2.0 \mu \mathrm{g} \mathrm{mL}^{-1}$. Similarly, the optimal concentration of the antiFLU-HRP was analyzed using a concentration range of $4.0-1.0 \mu \mathrm{g} \mathrm{mL}^{-1}$. Incubation for the immunocapture phase using the antiLAM-MP and with the antiLAMFLU was made at RT for $1 \mathrm{~h}$ with mixing at $750 \mathrm{rpm}$ while the antiFLU-HRP was incubated for $30 \mathrm{~min}$. Single washing with PBST supplemented with $0.1 \%$ casein was also performed at $750 \mathrm{rpm}$ prior to the addition of antibodies after the immunocapturing phase, and final washing of the reaction was done thrice prior to the addition of the substrate. TMB substrate reaction was performed under dark conditions for 30 min followed by the addition of $2.0 \mathrm{M}$ sulfuric acid prior to reading at $450 \mathrm{~nm}$.

\subsection{Magneto-actuated immunoassay for the detection of myco- bacterium in hemodialysis water}

Fig. 1 schematically represents the magneto-actuated immunoassay procedure in a 2-step sandwich immunoassay approach, using the optimized concentration of reagents as described above. Firstly, (A) the immunomagnetic separation was performed using the tailored antiLAM-MPs by incubation of $100 \mu \mathrm{L}$ of the antiLAM-MP $\left(0.075 \mathrm{mg} \mathrm{mL}^{-1}\right)$ with $100 \mu \mathrm{L}$ of the $M$.

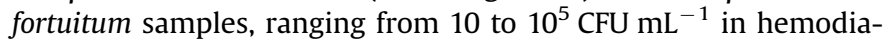
lysis water, at RT for $1 \mathrm{~h}$ while agitating at $750 \mathrm{rpm}$. After magnetic actuation of the microplates, the antiLAM-MPs with captured mycobacteria were washed once with PBST supplemented with $0.1 \%$ casein with agitation at $750 \mathrm{rpm}$ for $5 \mathrm{~min}$. (B) Thereafter, $100 \mu \mathrm{L}$ of the antiLAM-FLU $\left(1.0 \mu \mathrm{g} \mathrm{mL}^{-1}\right)$ was reacted at RT for $1 \mathrm{~h}$ with mixing at $750 \mathrm{rpm}$. (C) On the other hand, $100 \mu \mathrm{L}$ of the antiFLU-HRP $\left(4.0 \mu \mathrm{g} \mathrm{mL}^{-1}\right)$ was incubated for $30 \mathrm{~min}$ following a single wash performed as described above. Final washing of the reaction was done three times. (D) The optical readout at $450 \mathrm{~nm}$ was finally achieved by TMB substrate reaction performed under dark conditions for 30 min followed by the addition of $2.0 \mathrm{M}$ sulfuric acid. After each incubation or washing step, a 96-well magnet plate separator was positioned under the microtiter plate until pellet formation on the bottom corner, followed by supernatant separation.

The analytical performance of the magneto-actuated immunoassay was studied using hemodialysis water as matrix. Hemodialysis water was used since NTM are associated with infection arising from contamination of water in hemodialysis patients. The hemodialysis water was kindly provided by the Servei de Nefrologia, Hospital Parc Tauli Sabadell and was spiked with $M$. fortuitum ranging from 10 to $10^{5} \mathrm{CFU} \mathrm{mL} \mathrm{mL}^{-1}$ and a negative control was also prepared. In order to prepare a matrix closely similar to the original, with minimal TSB-0,025\% Tween 20 content, spiking was performed by subjecting $1 \mathrm{~mL}$ of the cultures in different concentrations $\left(10-10^{5} \mathrm{CFU} \mathrm{mL} \mathrm{m}^{-1}\right.$ ) prepared in TSB with $0,025 \%$ Tween 20 to centrifugation at $3500 \mathrm{~g}$ for $10 \mathrm{~min}$ [14] to harvest the mycobacteria. The volume of TSB-0,025\% Tween 20 was then reduced to $50 \mu \mathrm{L}$, and $950 \mu \mathrm{L}$ of sterile hemodialysis water was then added to prepare spiked samples which contain 95\% hemodialysis water. The samples were then analyzed using the optimized magneto-actuated immunoassay conditions to determine the LOD, as previously described.

The evaluation of the specificity of the magneto immunoassay was made using Escherichia coli and Pseudomonas aeruginosa. The system was challenged by analyzing cultures of $E$. coli and $P$. aeruginosa at $1.0 \times 10^{5} \mathrm{CFU} \mathrm{mL}^{-1}$ (OD 0.200). 

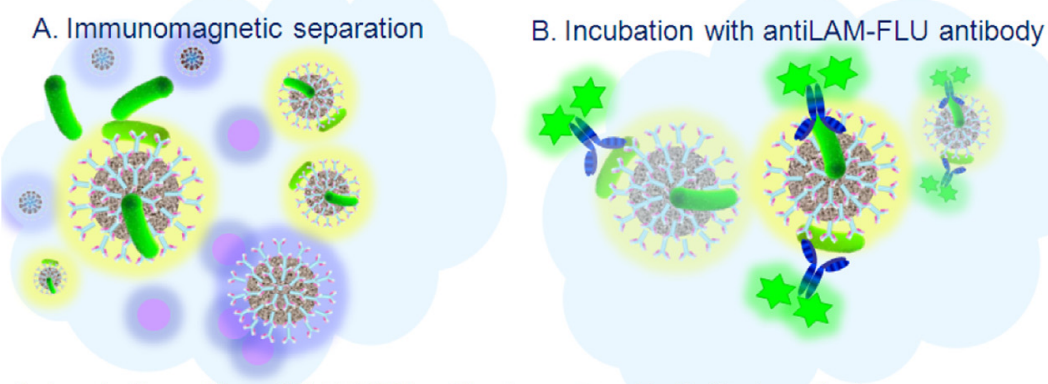

C. Incubation with antiFLU-HRP antibody

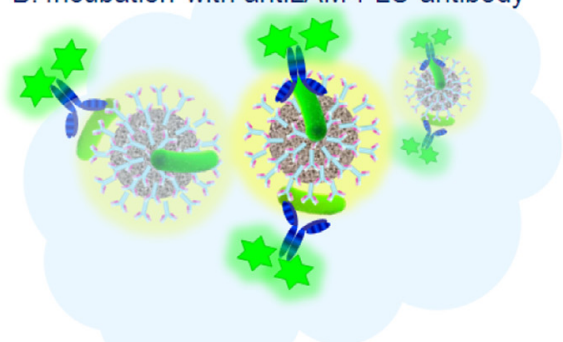

D. Optical readout

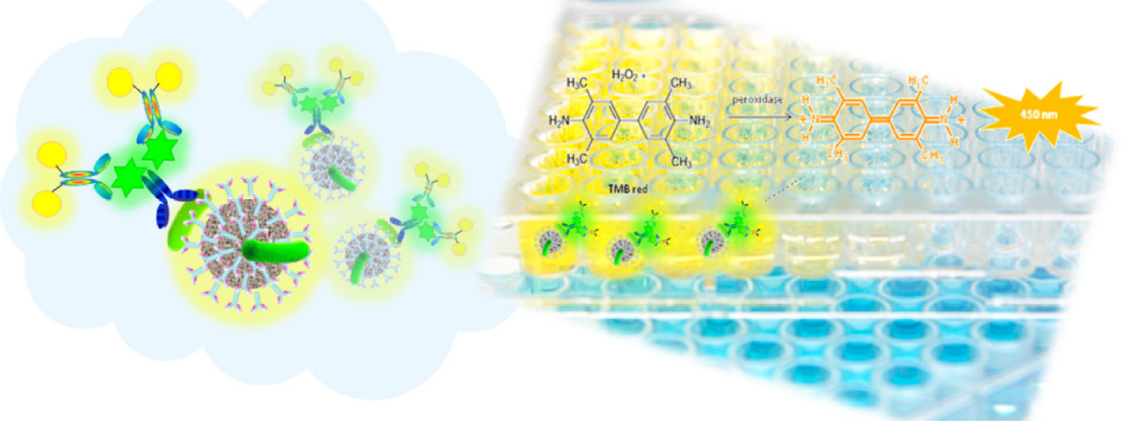

Fig. 1. Schematic representation of the magneto-actuated immunoassay procedure in a 2-step sandwich immunoassay approach.

\subsection{Biosafety considerations}

All experiments were performed in a Biosafety Class 2 environment required for the handling of $M$. fortuitum. All biological waste generated from the experiments were disposed in accordance to the local regulations for handling biohazards.

\section{Results and discussion}

\subsection{Immunoreactivity study of the antiLAM and antiLAM-FLU antibodies}

Fig. 2, panel A, shows the results of the antiLAM antibody immunoreactivity study, which clearly illustrate the ability of the antiLAM antibody to detect the LAM of M. fortuitum. This finding supports the suitability of the antiLAM antibody from guinea pig as a capture and labeling antibody to be used in a sandwich assay in the preparation of both the tailored antiLAM-MPs and the
antiLAM-FLU labeling antibody.

The antiLAM-FLU conjugates were prepared using the manufacturer's recommended protocol, as previously described. The purification antiLAM-FLU conjugates was performed by gel filtration chromatography. Desalting involves the chromatographic separation of macromolecules (in this instance the antiLAM-FLU antibody) from smaller molecules such as buffer components, salts and unreacted FITC labeling reagent. The number of desalting steps required to reduce non-specific adsorption was optimized, as shown in the supporting information section, Fig. S1, in order to achieve the higher signal-to-background radio. As observed in Fig. S1, 3X desalting steps following the FITC labeling reaction provides the best condition for removing the non-specific adsorption. This optimized desalting procedure was used in all cases during the preparation of the antiLAM-FLU conjugates.

On the other hand, the immunoreactivity of the antiLAM-FLU antibodies prepared against $M$. fortuitum was also evaluated in order to ensure both (i) that the antigen binding of the antibody is retained after the conjugation with fluorescein, and (ii) that the
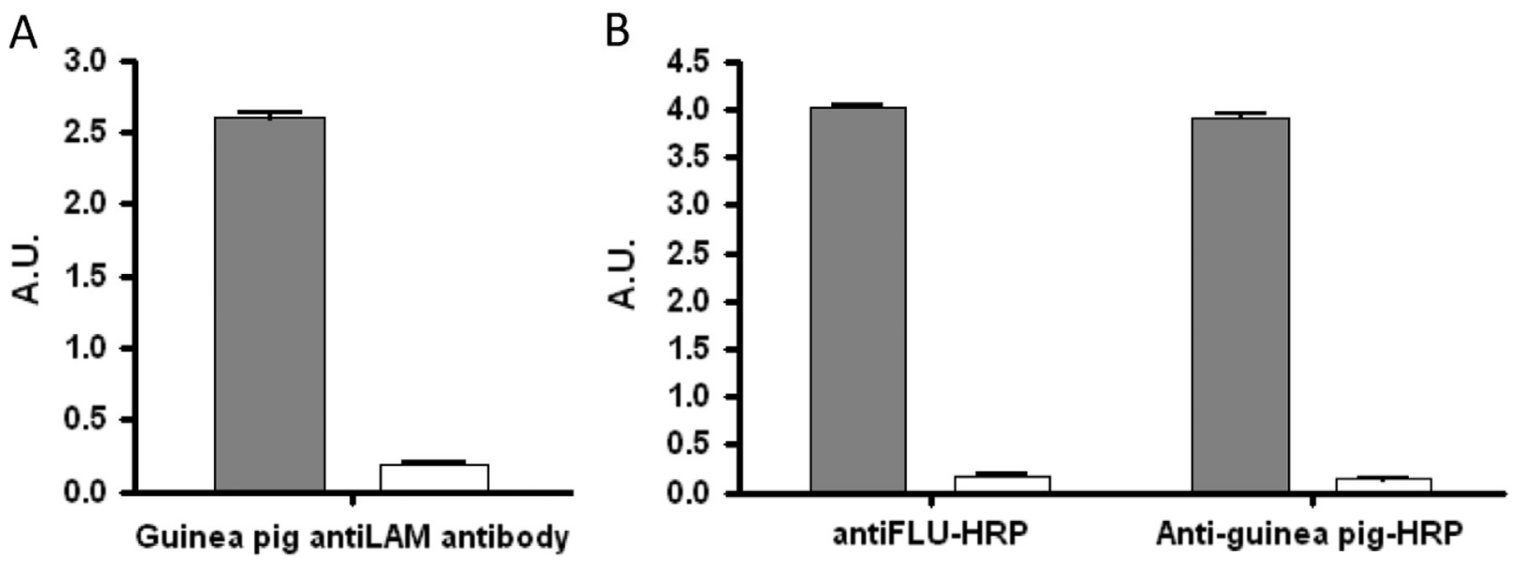

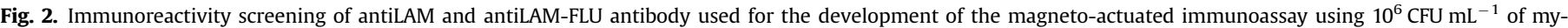

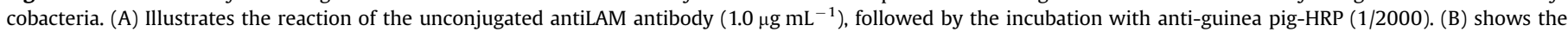

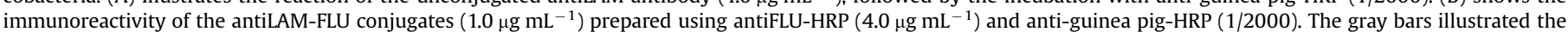
signal for $10^{6} \mathrm{CFU} \mathrm{mL}{ }^{-1}$, while the white bars show the background for $0 \mathrm{CFU} \mathrm{mL}{ }^{-1}$. Error bars illustrates the SD for $n=3$. 
antibody was accordingly modified with FITC and recognized with the antiFLU-HRP antibody. To achieve this task, two different secondary antibodies were used, respectively: anti-guinea pig-HRP and antiFLU-HRP antibody. As illustrated in Fig. 2, panel B, the binding sites of the antiLAM-FLU antibodies were not affected by the conjugation procedure, since they are able to react with the LAM of the mycobacteria adsorbed on the wells. The use of antiguinea pig-HRP secondary antibody in this experiment further validates that the observed signals in the ELISA were due to the binding of the antiLAM-FLU antibodies, while the use of antiFLUHRP antibody confirms that the antiLAM antibody was successfully modified with fluorescein and that this tag is appropriately recognized by the antiFLU-HRP antibody. Moreover, the signals observed were not due to the nonspecific adsorption of Fluorescein as the desalting procedure after conjugation of FITC was optimized to avoid background signals.

\subsection{Covalent immobilization of antiLAM antibody on magnetic particles and confocal microscopy study}

Using confocal fluorescence microscopy, the ability of the antiLAM modified MPs to capture the $M$. fortuitum was visualized following the staining of the mycobacterial DNA. As can be observed in Fig. 3, the antiLAM-MPs (in green) are capable of capturing the mycobacteria (stained in blue). For interpretation of the references to color, the reader is referred to the web version of this article. In addition, panel A of Fig. 3 shows the overall view of the immunocapturing reaction, illustrating the ability of the tailored antiLAM-MPs in capturing both small and larger aggregates of mycobacteria. Panel B of Fig. 3 further highlights the ability of the antiLAM-MPs to cover huge aggregates of the mycobacteria for immunomagnetic separation. This result confirms that the antiLAM antibody was immobilized on the tosyl-MPs and are capable of binding the exposed LAM binding sites on the surface of the mycobacterial cell wall.

\subsection{Optimization of the magneto-actuated immunoassay}

Fig. 4 shows the optimization of the reagents involved in the magneto-actuated immunoassay including the antiLAM-MPs, the antiLAM-FLU, and the antiFLU-HRP in order to achieve the higher signal-to-background radio as shown in the supporting information section, Fig. S2.

Results of the optimization illustrates that $100 \mu \mathrm{L}$ of $0.075 \mathrm{mg} \mathrm{ml}^{-1}$ antiLAM-MP provides the minimum concentration needed with low non-specific adsorption as illustrated in Fig. 4 panel A. On the other hand, low non-specific adsorption was observed when $100 \mu \mathrm{L}$ of the antiLAM-FLU at concentration of $1.0 \mu \mathrm{g} \mathrm{mL}^{-1}$ for the sandwich immunocapturing reaction is used as illustrated in Fig. 4 panel B. In terms of the antiFLU-HRP secondary antibody used to produce the enzymatic signals in the immunocapturing reaction, maximal absorption signal with low background is observed when $100 \mu \mathrm{L}$ of the antiFLU-HRP at $4.0 \mu \mathrm{g} \mathrm{mL}^{-1}$ is used in the reactions. This observation is further illustrated in Fig. 4 panel C. These optimized immunoreagent concentrations were used in order to develop the magneto-actuated immunoassay for $M$. fortuitum detection in hemodialysis water samples.

\subsection{Magneto-actuated immunoassay for the detection of myco- bacteria in hemodialysis water}

The optical response of the magneto-actuated immunoassay towards mycobacteria (from 0 to $10^{5} \mathrm{CFU} \mathrm{mL}^{-1}$ ) in hemodialysis water is shown in Fig. 5, panel A. Hemodialysis water were spiked with different amounts of $M$. fortuitum (quantified by classical culturing). The magneto-actuated immunoassay was fitted using a nonlinear regression (Four Parameter Logistic Equation- GraphPad Prism Software) with the value of $\left(R^{2}=0.9867\right)$, as shown in Fig. 5, panel B. The LOD was estimated by processing the negative control samples $(n=10)$ in hemodialysis water, obtaining a mean value of 0.175 A.U with a SD of 0.031 . The cut-off value was then determined with a one-tailed $t$ test at a 95\% confidence level, giving a value of 0.232 A.U. (shown in Fig. 5, panel A, as the dotted lines). Based on this, the LOD was thus found to be as low as $13 \mathrm{CFU} \mathrm{mL}^{-1}$, being the logistic range from 89 to $7 \times 10^{4} \mathrm{CFU} \mathrm{mL} \mathrm{mL}^{-1}$. The signal corresponding to the LOD is shown as a dotted red line in Fig. 5, panel B.

The ability of the detection system to specifically detect mycobacteria was further evaluated by analyzing samples of $E$. coli and $P$. aeruginosa at a concentration level of $1.0 \times 10^{5} \mathrm{CFU} \mathrm{mL}^{-1}$. It is expected that since the magneto immunoassay uses the LAM as target for antibody-mediated capture, non-LAM producing bacteria will not be detected. The experimental results are shown in
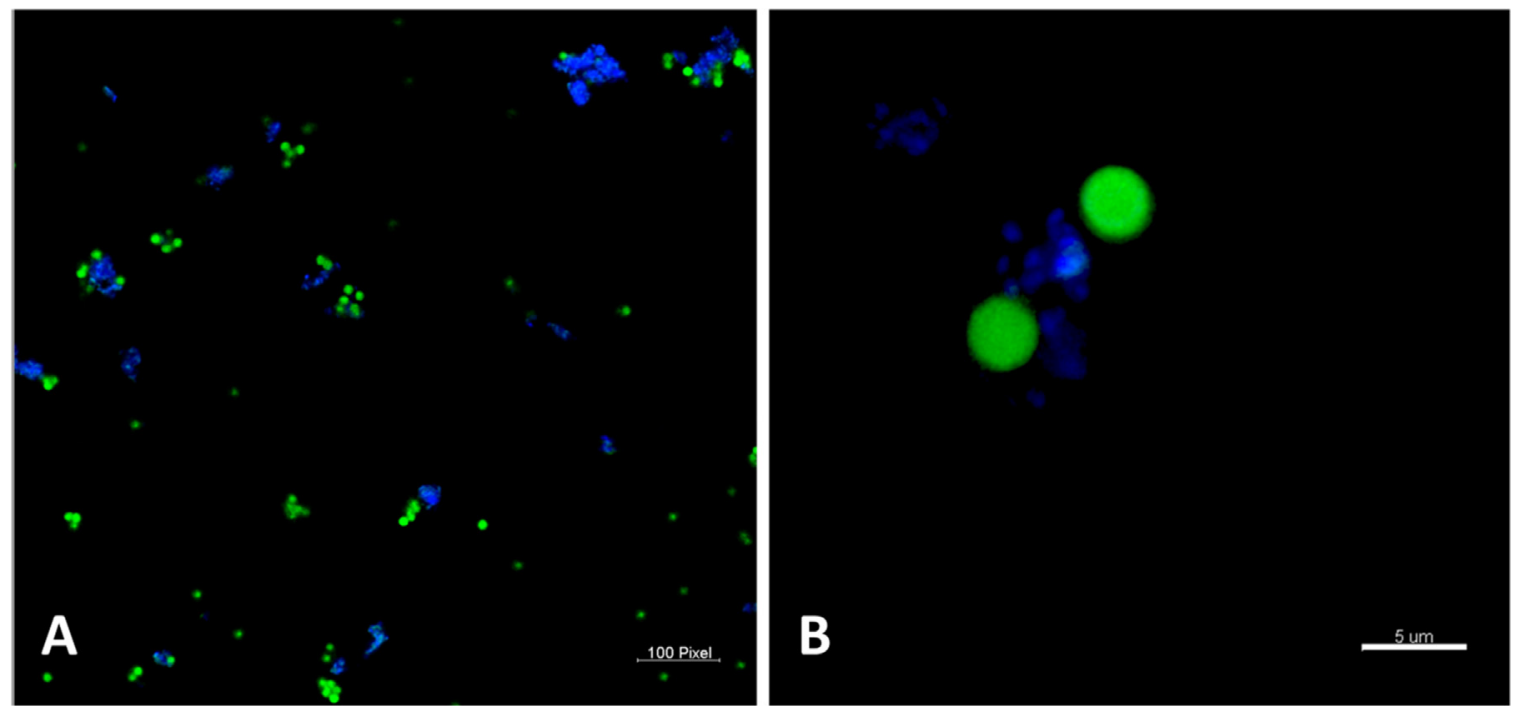

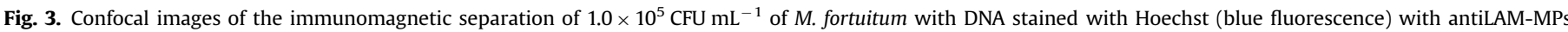

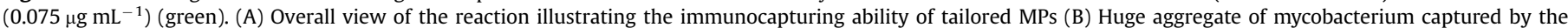
antiLAM-MPs. (For interpretation of the references to colour in this figure legend, the reader is referred to the web version of this article). 

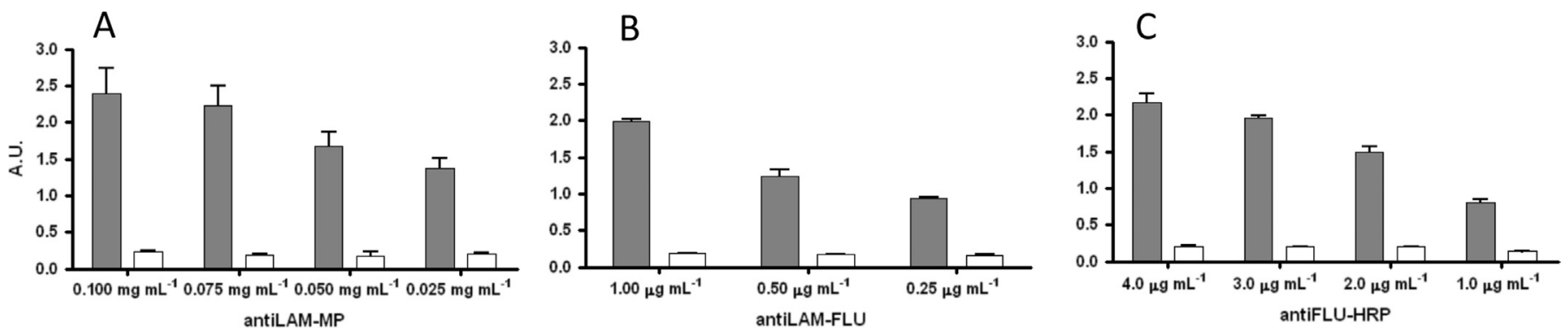

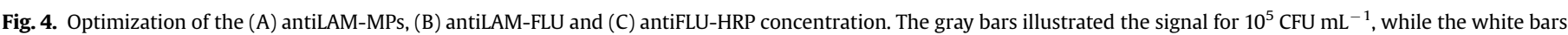
shows the background for $0 \mathrm{CFU} \mathrm{mL} \mathrm{m}^{-1}$. Error bars illustrates SD for $n=3$.
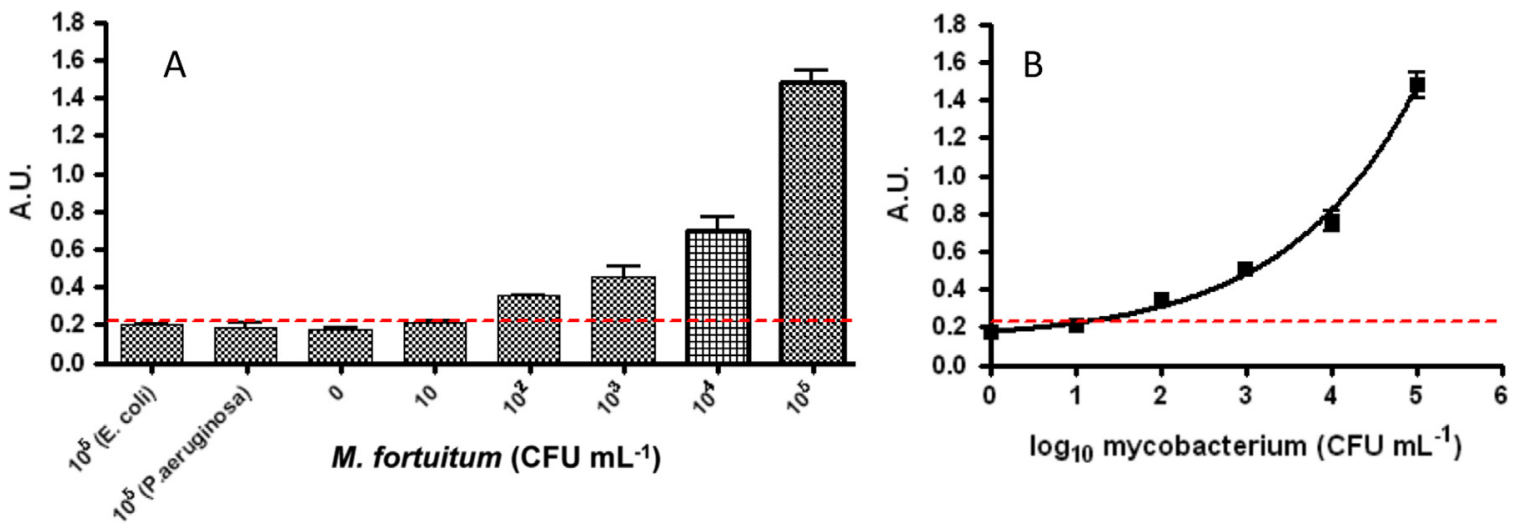

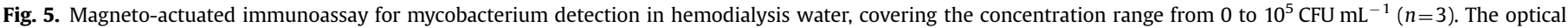

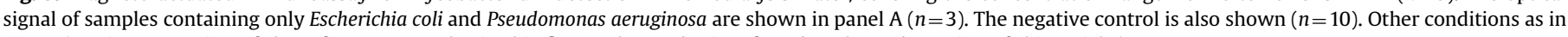
Fig. 4. (For interpretation of the references to color in this figure, the reader is referred to the web version of this article.)

Fig. 5, panel A. The samples containing $E$. coli and $P$. aeruginosa showed mean optical values of 0.203 and 0.184 A.U., respectively, lower than the cut-off value of 0.232 A.U. (shown in Fig. 5, panel A, as the dotted lines). These results illustrate specific detection of $M$. fortuitum since optical signals containing $0 \mathrm{CFU} \mathrm{mL} \mathrm{m}^{-1}$ of the mycobacteria (negative control) was found to be comparable when $E$. coli and $P$. aeruginosa containing samples were analyzed, suggesting a good specificity of the assay for detecting mycobacterium.

\section{Conclusions}

In this study, we present a rapid magneto-actuated immunoassay for the detection of mycobacteria in hemodialysis water. The immunoassay relies on the use of tailored antiLAM-MPs capable of binding LAM found in the surface of the mycobacteria, and the use of an antiFLU-HRP secondary antibody employed in an indirect sandwich immunoassay approach. The use of antiLAMMPs enables pre-concentration of the mycobacteria throughout immunomagnetic separation, including immunological reaction and magnetic actuation. The magneto-actuated immunoassay showed a LOD of $13 \mathrm{CFU} \mathrm{mL} \mathrm{m}^{-1}$ of mycobacteria in hemodialysis water, providing a sensitive method but by using instrumentation available in low-resource settings laboratories and requiring lowmaintenance, as is the case of a microplate reader operated by filters. With the increasing cases of patients with ESRD undergoing hemodialysis [1] and isolation of rapid growing environmental mycobacteria from dialysis related peritonitis [4,5] , a rapid and sensitive method for detecting the presence of mycobacteria in hemodialysis fluid could provide basis for infection control measures. The magneto-actuated immunoassay presented in this study illustrates a robust method capable of detecting levels of mycobacteria that is within the allowable viable colony counts in dialysate fluid ( $>200 \mathrm{CFU} \mathrm{mL}^{-1}$ ) [15]. Although the indirect ELISA included a further incubation step, it provides as a main advantage a higher sensitivity than the direct counterpart, since the signal is amplified. The fluorescein groups attached on the primary antiLAM antibodies will further react with the secondary antibody conjugated with the optical reporter (polyclonal antiFLU-HRP), carrying many HRP per molecule of antibody. Moreover, the high turnover of the enzyme provides further amplification of the signal, since many molecules of the optical active product are produce per minute. One of the main disadvantages of the conventional method of detecting mycobacteria in hemodialysis fluid, which is mainly addressed by the magneto-actuated immunoassay, is the time required to identify the presence of mycobacteria in dialysis fluid samples. Combined with the outstanding performance of the antiLAM-MP to capture mycobacteria, the present system only requires a total of $3 \mathrm{~h}$ incubation time for the immunocapturing, secondary antibody and substrate reaction to specifically detect mycobacteria as compared to the conventional method. Conventionally, mycobacteria from dialysis fluid are isolated using membrane filtration and spread plate technique requiring minimum of 3-7 days incubation to initially isolate rapid growing mycobacteria.

\section{Acknowledgment}

Financial support from the Ministry of Economy and Competitiveness (MINECO), Madrid (BIO2013-41242-R), and Fundação de Amparo à Pesquisa do Estado de São Paulo (FAPESP), Brazil (process 2008/10449-7) are acknowledged. RBJr acknowledges the Erasmus Mundus MAHEVA scholarship provided by the EU Commission for his stay in UAB and the Philippine Council for Health Research and Foundation (PCHRD), Department of Science and Technology (DOST) for supporting the academic phase of his 
doctoral degree at the College of Public Health, University of the Philippines Manila, Philippines.

\section{Appendix A. Supplementary material}

Supplementary data associated with this article can be found in the online version at http://dx.doi.org/10.1016/j.talanta.2016.02.041.

\section{References}

[1] S.L. White, S.J. Chadban, S. Jan, J.R. Chapman, A. Cass, How can we achieve global equity in provision of renal replacement therapy? Bull. World Health Organ. 86 (2008) 229-237, http://dx.doi.org/10.2471/BLT.07.041715.

[2] M.K.-H. Tong, W. Wang, T.-H. Kwan, L. Chan, T.-C. Au, Water treatment for hemodialysis, Hong Kong J. Nephrol. 3 (2001) 7-14, http://dx.doi.org/10.1016/ S1561-5413(09)60050-8.

[3] S. Hess, V. Bren, Essential components of an infection prevention program for outpatient hemodialysis centers, Semin. Dial. 26 (2013) 384-398, http://dx. doi.org/10.1111/sdi.12102.

[4] F.G. Sartori, L.F. Leandro, L.B. Montanari, M.G.M. De Souza, R.H. Pires, D.N. Sato, et al., Isolation and identification of environmental mycobacteria in the waters of a hemodialysis center, Curr. Microbiol. 67 (2013) 107-111, http://dx.doi.org/ 10.1007/s00284-013-0341-6.

[5] M. Kunin, A. Knecht, E.J. Holtzman, Mycobacterium chelonae peritonitis in peritoneal dialysis. Literature review, Eur. J. Clin. Microbiol. Infect. Dis. 33 (2014) 1267-1271, http://dx.doi.org/10.1007/s10096-014-2079-x.

[6] L. Hall-Stoodley, H. Lappin-Scott, Biofilm formation by the rabidly growing mycobacterial species Mycobacterium fortuitum, FEMS Microbiol. Lett. 168
(1998) 77-84

[7] J. Esteban, N.Z Martín-de-Hijas, TJ. Kinnari, G. Ayala, R. Fernández-Roblas, I. Gadea, Biofilm development by potentially pathogenic non-pigmented rapidly growing mycobacteria, BMC Microbiol. 8 (2008) 184, http://dx.doi.org/ 10.1186/1471-2180-8-184.

[8] R. Nystrand, Microbiology of water and fluids for hemodialysis, J. Chin. Med. Assoc. 71 (2008) 223-229, http://dx.doi.org/10.1016/S1726-4901(08)70110-2.

[9] D. Brandao, S. Liébana, S. Campoy, S. Alegret, M. Isabel Pividori, Immunomagnetic separation of salmonella with tailored magnetic micro and nanocarriers. A comparative study, Talanta 143 (2015) 198-204, http://dx.doi org/10.1016/j.talanta.2015.05.035.

[10] S. Liébana, A. Lermo, S. Campoy, M.P. Cortés, S. Alegret, M.I. Pividori, Rapid detection of Salmonella in milk by electrochemical magneto-immunosensing, Biosens. Bioelectron. 25 (2009) 510-513, http://dx.doi.org/10.1016/j. bios.2009.07.022.

[11] K.Y. Lien, L.Y. Hung, T.B. Huang, Y.C. Tsai, H.Y. Lei, G.B. Lee, Rapid detection of influenza A virus infection utilizing an immunomagnetic bead-based microfluidic system, Biosens. Bioelectron. 26 (2011) 3900-3907.

[12] J. Kim, J. Lee, K.I. Lee, T.P. Park, H.J. Kim, J. Lee, Rapid monitoring of CFP-10 during cultura of Mycobacterium tuberculosis by using a magnetophoretic immunoassay, Sens. Actuator B 177 (2013) 327-333.

[13] J.M. Martin, P.F. Riska, B.R. Bloom, A. Glatman-freedman, J.M. Martin, P.F. Riska et al., Monoclonal antibodies to surface antigens of Mycobacterium tuberculosis and their use in a modified enzyme-linked immunosorbent spot assay for detection of mycobacteria . These include: Monoclonal Antibodies to Surface Antigens of Mycobacterium tuberculo, 1996.

[14] C.A. Speer, M.C. Scott, J.P. Bannantine, W. Ray, Y. Mori, R.H. Whitlock, et al., A novel enzyme-linked immunosorbent assay for diagnosis of Mycobacterium avium subsp. paratuberculosis Infections (Johne's disease) in cattle, Clin. Vaccine Immunol. 13 (2006) 535-540, http://dx.doi.org/10.1128/CVI.13.5.535.

[15] M.J. Arduino, L. a Bland, S.M. Aguero, L. Carson, M. Ridgeway, M.S. Favero, Comparison of microbiologic assay methods for hemodialysis fluids, J. Clin. Microbiol. 29 (1991) 592-594. 